\title{
A Characterization of Hardy Spaces Associated with Certain Schrödinger Operators
}

\author{
Jacek Dziubański • Jacek Zienkiewicz
}

Received: 6 June 2013 / Accepted: 4 March 2014 / Published online: 11 April 2014

(C) Springer Science+Business Media Dordrecht 2014

\begin{abstract}
Let $\left\{K_{t}\right\}_{t>0}$ be the semigroup of linear operators generated by a Schrödinger operator $-L=\Delta-V(x)$ on $\mathbb{R}^{d}, d \geq 3$, where $V(x) \geq 0$ satisfies $\Delta^{-1} V \in L^{\infty}$. We say that an $L^{1}$-function $f$ belongs to the Hardy space $H_{L}^{1}$ if the maximal function $\mathcal{M}_{L} f(x)=\sup _{t>0}\left|K_{t} f(x)\right|$ belongs to $L^{1}\left(\mathbb{R}^{d}\right)$. We prove that the operator $(-\Delta)^{1 / 2} L^{-1 / 2}$ is an isomorphism of the space $H_{L}^{1}$ with the classical Hardy space $H^{1}\left(\mathbb{R}^{d}\right)$ whose inverse is $L^{1 / 2}(-\Delta)^{-1 / 2}$. As a corollary we obtain that the space $H_{L}^{1}$ is characterized by the Riesz transforms $R_{j}=\frac{\partial}{\partial x_{j}} L^{-1 / 2}$.
\end{abstract}

Keywords Hardy spaces $\cdot$ Schrödinger operators

Mathematics Subject Classifications (2010) 42B30 · 35J10 • 42B35

\section{Introduction and Statement of the Result}

Let $\mathcal{L}=-\Delta+\mathcal{V}(x)$ be a Schrödinger operator on $\mathbb{R}^{d}$, where $\mathcal{V}(x)$ is a nonnegative locally integrable potential and let $\left\{T_{t}\right\}_{t>0}$ be the semigroup generated by $-\mathcal{L}$. The action of the semigroup is given by the Feynman-Kac formula

$$
T_{t} f(x)=E^{x}\left(e^{-\int_{0}^{t} \mathcal{V}\left(X_{s}\right) d s} f\left(X_{t}\right)\right),
$$

The research was supported by Polish funds for sciences, grants: DEC-2012/05/B/ST1/00672 and DEC-2012/05/B/ST1/00692 from Narodowe Centrum Nauki.

J. Dziubański $(\bowtie) \cdot$ J. Zienkiewicz

Instytut Matematyczny, Uniwersytet Wrocławski, Pl. Grunwaldzki 2/4, 50-384 Wrocław, Poland

e-mail: jdziuban@math.uni.wroc.pl

J. Zienkiewicz

e-mail: zenek@math.uni.wroc.pl 
where $X_{t}$ is a Brownian motion associated with the heat semigroup $P_{t}=e^{t \Delta}$ (see, e.g., Chapter $\mathrm{V}$ of [18]). Let $T_{t}(x, y)$ denote the integral kernel of the semigroup $\left\{T_{t}\right\}_{t>0}$. Since $\mathcal{V}(x)$ is non-negative, Eq. 1.1 implies that

$$
0 \leq T_{t}(x, y) \leq(4 \pi t)^{-d / 2} e^{-|x-y|^{2} / 4 t}=: P_{t}(x-y) .
$$

It easily follows from Eq. 1.2 that the maximal function

$$
\mathcal{M}_{\mathcal{L}} f(x)=\sup _{t>0}\left|T_{t} f(x)\right|
$$

is a bounded operator on $L^{p}\left(\mathbb{R}^{d}\right)$ for $1<p \leq \infty$ and of weak-type (1.1).

The real Hardy space $H_{\mathcal{L}}^{1}$ associated with $\mathcal{L}$ is defined as

$$
H_{\mathcal{L}}^{1}=\left\{f \in L^{1}\left(\mathbb{R}^{d}\right): \mathcal{M}_{\mathcal{L}} f \in L^{1}\left(\mathbb{R}^{d}\right)\right\}
$$

with the norm

$$
\|f\|_{H_{\mathcal{L}}^{1}}=\left\|\mathcal{M}_{\mathcal{L}} f\right\|_{L^{1}\left(\mathbb{R}^{n}\right)} .
$$

Let us note that the Hardy space $H_{\mathcal{L}}^{1}$ coincides with the Hardy spaces $H_{\mathcal{L} \text {, max, } h}^{1}\left(\mathbb{R}^{d}\right)$ considered in Hofmann et al. [14, Chapters 7 and 8], where $H_{\mathcal{L}, \max , h}^{1}\left(\mathbb{R}^{d}\right)$ is defined as the completion of the space

$$
\left\{f \in L^{2}\left(\mathbb{R}^{d}\right): \mathcal{M}_{\mathcal{L}} f \in L^{1}\left(\mathbb{R}^{d}\right)\right\}
$$

in the norm Eq. 1.5. We shall present a proof of this fact using standard arguments in the Appendix.

In the present paper we consider the semigroup $\left\{K_{t}\right\}_{t>0}$ of linear operators on $\mathbb{R}^{d}, d \geq 3$, generated by a Schrödinger operator $-L=\Delta-V(x)$, where $V(x)$ is a non-negative locally integrable function which satisfies

$$
\Delta^{-1} V(x)=-c_{d} \int_{\mathbb{R}^{d}} \frac{1}{|x-y|^{d-2}} V(y) d y \in L^{\infty}\left(\mathbb{R}^{d}\right) .
$$

Let $K_{t}(x, y)$ denote the integral kernel of the semigroup $\left\{K_{t}\right\}_{t>0}$. Clearly, the upper Gaussian bounds (1.2) hold for $K_{t}(x, y)$. It is known, see [17], that for $V(x) \geq 0$ the condition (1.6) is equivalent to the lower Gaussian bounds for $K_{t}(x, y)$, that is, there are $c, C>0$ such that

$$
c t^{-d / 2} e^{-C|x-y|^{2} / t} \leq K_{t}(x, y) .
$$

The Hardy spaces $H_{L}^{1}$ associated with Schrödinger operators with nonnegative potentials satisfying Eq. 1.6 were studied in [12]. It was proved that the map $f(x) \mapsto w(x) f(x)$ is an isomorphism of $H_{L}^{1}$ onto the classical Hardy space $H^{1}\left(\mathbb{R}^{d}\right)$, where

$$
w(x)=\lim _{t \rightarrow \infty} \int K_{t}(x, y) d y,
$$

which in particular means that

$$
\|f w\|_{H^{1}\left(\mathbb{R}^{d}\right)} \sim\|f\|_{H_{L}^{1}}
$$

see [12, Theorem 1.1]. The function $w(x)$ is $L$-harmonic, that is, $K_{t} w=w$, and satisfies $0<\delta \leq w(x) \leq 1$.

Let us remark that the classical real Hardy space $H^{1}\left(\mathbb{R}^{d}\right)$ can be thought as the space $H_{L}^{1}$ associated with the classical heat semigroup $e^{t \Delta}$, that is, $L=-\Delta+V$ with $V \equiv 0$ in this case. Obviously, the constant functions are the only bounded harmonic functions for $\Delta$.

The present paper is a continuation of [12]. Our goal is to study the mappings

$$
L^{1 / 2}(-\Delta)^{-1 / 2} \text { and }(-\Delta)^{1 / 2} L^{-1 / 2}
$$


which turn out to be bounded on $L^{1}\left(\mathbb{R}^{d}\right)$ (see Lemma 2.6). Our main result is the following theorem, which states another characterization of $H_{L}^{1}$.

Theorem 1.10 Assume that $L=-\Delta+V(x)$ is a Schrödinger operator on $\mathbb{R}^{d}, d \geq 3$, with a locally integrable non-negative potential $V(x)$ satisfying Eq. 1.6. Then the mapping $f \mapsto(-\Delta)^{1 / 2} L^{-1 / 2} f$ is an isomorphism of $H_{L}^{1}$ onto the classical Hardy space $H^{1}\left(\mathbb{R}^{d}\right)$, that is, there is a constant $C>0$ such that

$$
\begin{gathered}
\left\|(-\Delta)^{1 / 2} L^{-1 / 2} f\right\|_{H^{1}\left(\mathbb{R}^{d}\right)} \leq C\|f\|_{H_{L}^{1}}, \\
\left\|L^{1 / 2}(-\Delta)^{-1 / 2} f\right\|_{H_{L}^{1}} \leq C\|f\|_{H^{1}\left(\mathbb{R}^{d}\right)} .
\end{gathered}
$$

As a corollary we immediately obtain the following Riesz transform characterization of $H_{L}^{1}$.

Corollary 1.13 Under the assumptions of Theorem 1.10 an $L^{1}$-function $f$ belongs to the space $H_{L}^{1}$ if and only if $R_{j} f=\frac{\partial}{\partial x_{j}} L^{-1 / 2} f$ belong to $L^{1}\left(\mathbb{R}^{d}\right)$ for $j=1,2, \ldots, d$. Moreover, there is a constant $C>0$ such that

$$
C^{-1}\|f\|_{H_{L}^{1}} \leq\|f\|_{L^{1}\left(\mathbb{R}^{d}\right)}+\sum_{j=1}^{d}\left\|R_{j} f\right\|_{L^{1}\left(\mathbb{R}^{d}\right)} \leq C\|f\|_{H_{L}^{1}} .
$$

Example 1 It is not hard to see that if for a function $V(x) \geq 0$ defined on $\mathbb{R}^{d}, d \geq 3$, there is $\varepsilon>0$ such that $V \in L^{d / 2-\varepsilon}\left(\mathbb{R}^{d}\right) \cap L^{d / 2+\varepsilon}\left(\mathbb{R}^{d}\right)$, then $V$ satisfies Eq. 1.6.

Example 2 Assume that Eq. 1.6 holds for a function $V: \mathbb{R}^{d} \rightarrow[0, \infty), d \geq 3$. Then $V\left(x_{1}, x_{2}\right):=V\left(x_{1}\right)$ defined on $\mathbb{R}^{d} \times \mathbb{R}^{n}, n \geq 1$, fulfils Eq. 1.6.

The reader interested in other results concerning Hardy spaces associated with semigroups of linear operators, and in particular semigroups generated by Schrödinger operators, is referred to $[1,2,6-10,14]$.

\section{Boundedness on $L^{1}$}

We define the operators:

$$
\begin{aligned}
(-\Delta)^{-1} f(x) & =\int_{0}^{\infty} P_{t} f(x) d t=c_{d} \int \frac{f(y)}{|x-y|^{d-2}} d y=: \int \Gamma_{0}(x-y) f(y) d y, \\
L^{-1} f(x) & =\int_{0}^{\infty} K_{t} f(x) d t=: \int \Gamma(x, y) f(y) d y, \\
(-\Delta)^{-1 / 2} f & =c_{1} \int_{0}^{\infty} P_{t} f \frac{d t}{\sqrt{t}}=c_{d}^{\prime} \int \frac{1}{|x-y|^{d-1}} f(y) d y=: \int \widetilde{\Gamma}_{0}(x-y) f(y) d y, \\
L^{-1 / 2} f & =c_{1} \int_{0}^{\infty} K_{t} f \frac{d t}{\sqrt{t}}=: \int \widetilde{\Gamma}(x, y) f(y) d y,
\end{aligned}
$$

where $c_{1}=\Gamma(1 / 2)^{-1}$. Clearly, by Eq. 1.2,

$$
0 \leq \tilde{\Gamma}(x, y) \leq c_{d}^{\prime}|x-y|^{-d+1}, \quad 0<\Gamma(x, y) \leq c_{d}|x-y|^{-d+2} .
$$


The perturbation formula asserts that

$$
\begin{aligned}
P_{t}(x-y) & =K_{t}(x, y)+\int_{0}^{t} \int P_{t-s}(x-z) V(z) K_{s}(z, y) d z d s \\
& =K_{t}(x, y)+\int_{0}^{t} \int K_{t-s}(x, z) V(z) P_{s}(z-y) d z d s .
\end{aligned}
$$

Multiplying the second inequality in Eq. 2.2 by $w(x)$ and integrating with respect to $d x$ we get

$$
\int P_{t}(x-y) w(x) d x=w(y)+\int_{\mathbb{R}^{d}} \int_{0}^{t} w(z) V(z) P_{s}(z, y) d s d x,
$$

since $w$ is $L$-harmonic. The left-hand side of Eq. 2.3 tends to a harmonic function, which is bounded from below by $\delta$ and above by 1 , as $t$ tends to infinity. Thus there is a constant $0<c_{w} \leq 1$ such that

$$
c_{w}=w(y)+\int_{\mathbb{R}^{d}} w(z) V(z) \Gamma_{0}(z-y) d z .
$$

Similarly, integrating the first equation in Eq. 2.2 with respect to $x$ and taking limit as $t$ tends to infinity, we obtain

$$
1=w(y)+\int_{\mathbb{R}^{d}} V(z) \Gamma(z, y) d z .
$$

For a reasonable function $f$ the following operators are well defined in the sense of distributions:

$$
\begin{aligned}
& (-\Delta)^{1 / 2} f=c_{2} \int_{0}^{\infty}\left(P_{t} f-f\right) \frac{d t}{t^{3 / 2}}, c_{2}=\Gamma(-1 / 2)^{-1}, \\
& L^{1 / 2}=c_{2} \int_{0}^{\infty}\left(K_{t} f-f\right) \frac{d t}{t^{3 / 2}} .
\end{aligned}
$$

Lemma 2.6 There is a constant $C>0$ such that

$$
\begin{aligned}
& \left\|(-\Delta)^{1 / 2} L^{-1 / 2} f\right\|_{L^{1}} \leq C\|f\|_{L^{1}}, \\
& \left\|L^{1 / 2}(-\Delta)^{-1 / 2} f\right\|_{L^{1}} \leq C\|f\|_{L^{1}} .
\end{aligned}
$$

Proof From the perturbation formula (2.2) we have

$$
\begin{aligned}
& (-\Delta)^{1 / 2} L^{-1 / 2} f(x)=c_{2} \int_{0}^{\infty}\left(P_{t}-I\right) L^{-1 / 2} f(x) \frac{d t}{t^{3 / 2}} \\
& =c_{2} \int_{0}^{\infty}\left(P_{t}-K_{t}\right) L^{-1 / 2} f(x) \frac{d t}{t^{3 / 2}}+c_{2} \int_{0}^{\infty}\left(K_{t}-I\right) L^{-1 / 2} f(x) \frac{d t}{t^{3 / 2}} \\
& =c_{2} \int_{0}^{\infty} \int_{0}^{t} \iint P_{t-s}(x-z) V(z) K_{s}(z, y) L^{-1 / 2} f(y) d y d z d s \frac{d t}{t^{3 / 2}}+f(x) .
\end{aligned}
$$

Consider the integral kernel $W(x, u)$ of the operator

$$
f \mapsto \int_{0}^{\infty} \int_{0}^{t} \iint P_{t-s}(x-z) V(z) K_{s}(z, y) L^{-1 / 2} f(y) d y d z d s \frac{d t}{t^{3 / 2}},
$$


that is,

$$
W(x, u)=\int_{0}^{\infty} \int_{0}^{t} \iint P_{t-s}(x-z) V(z) K_{s}(z, y) \tilde{\Gamma}(y, u) d y d z d s \frac{d t}{t^{3 / 2}}
$$

Clearly $0 \leq W(x, u)$. Integration of $W(x, u)$ with respect to $d x$ leads to

$$
\begin{aligned}
\int W(x, u) d x & =\int_{0}^{\infty} \int_{0}^{t} \iint V(z) K_{s}(z, y) \tilde{\Gamma}(y, u) d y d z d s \frac{d t}{t^{3 / 2}} \\
& =2 \int_{0}^{\infty} \iint V(z) K_{s}(z, y) \tilde{\Gamma}(y, u) d y d z \frac{d s}{\sqrt{s}} \\
& \leq 2 c_{1}^{-1} \iint V(z) \widetilde{\Gamma}(z, y) \widetilde{\Gamma}(y, u) d y d z \\
& =2 c_{1}^{-1} \int V(z) \Gamma(z, u) d z .
\end{aligned}
$$

Using Eq. 2.1 we see that $\int W(x, u) d x \leq 2 c_{1}^{-1}\left\|\Delta^{-1} V\right\|_{L^{\infty}}$, which completes the proof of Eq. 2.7. The proof of Eq. 2.8 goes in the same way. We skip the details.

We finish this section by proving the following two lemmas, which will be used in the sequel.

Lemma 2.11 Assume that $f \in L^{1}\left(\mathbb{R}^{d}\right)$. Then

$$
\int(-\Delta)^{1 / 2} L^{-1 / 2} f(x) d x=\int f(x) w(x) d x .
$$

Proof From Eqs. 2.9 and 2.10 we conclude that

$$
\begin{aligned}
\int(-\Delta)^{1 / 2} L^{-1 / 2} f(x) d x & =c_{2} \iint W(x, u) f(u) d u d x+\int f(x) d x \\
& =2 c_{2} c_{1}^{-1} \int V(z) \Gamma(z, u) f(u) d z d u+\int f(x) d x \\
& =\int(w(u)-1) f(u) d u+\int f(x) d x,
\end{aligned}
$$

where in the last equality we have used Eq. 2.5.

Lemma 2.13 Assume that $f \in L^{1}\left(\mathbb{R}^{d}\right)$. Then

$$
\int\left(L^{1 / 2}(-\Delta)^{-1 / 2} f\right)(x) w(x) d x=c_{w} \int f(x) d x .
$$


Proof The proof is similar to that of Lemma 2.11. Indeed, by the perturbation formula (2.2) we have

$$
\begin{array}{r}
\int\left(L^{1 / 2}(-\Delta)^{-1 / 2} f\right)(x) w(x) d x \\
\left.=c_{2} \iint_{0}^{\infty}\left(K_{t}-P_{t}\right)\left((-\Delta)^{-1 / 2}\right) f\right)(x) \frac{d t}{t^{3 / 2}} w(x) d x \\
\left.+c_{2} \iint_{0}^{\infty}\left(P_{t}-I\right)\left((-\Delta)^{-1 / 2}\right) f\right)(x) \frac{d t}{t^{3 / 2}} w(x) d x \\
=-c_{2} \iint_{0}^{\infty} \int_{0}^{t} \iint w(x) K_{t-s}(x, z) V(z) \\
\times P_{S}(z-y)\left((-\Delta)^{-\frac{1}{2}} f\right)(y) d y d z d s \frac{d t}{t^{3 / 2}} d x \\
+\int w(x) f(x) d x \\
=-c_{2} \int_{0}^{\infty} \int_{0}^{t} \int_{\mathbb{R}^{d}} \int_{\mathbb{R}^{d}} w(z) V(z) P_{s}(z-y)\left((-\Delta)^{-1 / 2} f\right)(y) \\
\times d y d z d s \frac{d t}{t^{3 / 2}}+\int w(x) f(x) d x,
\end{array}
$$

where in the last equality we have used that $w$ is $L$-harmonic. Integrating with respect to $d t$ and then with respect to $d s$ yields

$$
\begin{aligned}
\int & \left(L^{1 / 2}(-\Delta)^{-1 / 2} f\right)(x) w(x) d x \\
& =-\frac{2 c_{2}}{c_{1}} \iint w(z) V(z) \widetilde{\Gamma}_{0}(z-y)\left((-\Delta)^{-1 / 2} f\right)(y) d y d z+\int f(x) w(x) d x \\
& =\int w(z) V(z) \Gamma_{0}(z-u) f(u) d u d z+\int f(x) w(x) d x \\
& =\int c_{w} f(x) d x-\int w(y) f(y) d y+\int f(x) w(x) d x
\end{aligned}
$$

where in the last equality we have used Eq. 2.4.

\section{Atoms and Molecules}

Fix $1<q \leq \infty$. We say that a function $a$ is an $(1, q, w)$-atom if there is a ball $B \subset \mathbb{R}^{d}$ such that supp $a \subset B,\|a\|_{L^{q}\left(\mathbb{R}^{d}\right)} \leq|B|^{\frac{1}{q}-1}, \int a(x) w(x) d x=0$. The atomic norm $\|f\|_{H^{1} \text { at }, q, w}$ is defined by

$$
\|f\|_{H_{\mathrm{at}, q, w}^{1}}=\inf \left\{\sum_{j=1}^{\infty}\left|\lambda_{j}\right|\right\},
$$

where the infimum is taken over all representations $f=\sum_{j=1}^{\infty} \lambda_{j} a_{j}$, where $\lambda_{j} \in \mathbb{C}, a_{j}$ are $(1, q, w)$-atoms.

Clearly, if $w_{0}(x) \equiv 1$, then the $\left(1, q, w_{0}\right)$-atoms coincide with the classical $(1, q)$-atoms for the Hardy space $H^{1}\left(\mathbb{R}^{d}\right)$, which can be thought as $H_{-\Delta}^{1}$.

As a direct consequence of Theorem 1.1 of [12] (see Eq. 1.9) and the results about atomic decompositions of the classical real Hardy spaces (see, e.g., $[3,15,19]$ ), we obtain that 
the space $H_{L}^{1}$ admits atomic decomposition into $(1, q, w)$-atoms, that is, there is a constant $C_{q}>0$ such that

$$
C_{q}^{-1}\|f\|_{H_{\mathrm{at}, q, w}^{1}} \leq\|f\|_{H_{L}^{1}} \leq C_{q}\|f\|_{H_{\mathrm{at}, q, w}^{1}} .
$$

Let $\varepsilon>0,1<q<\infty$. We say that a function $b$ is a $(1, q, \varepsilon, w)$-molecule associated with a ball $B=B\left(x_{0}, r\right)$ if

$$
\left(\int_{B}|b(x)|^{q} d x\right)^{\frac{1}{q}} \leq|B|^{\frac{1}{q}-1}, \quad\left(\int_{2^{k} B \backslash 2^{k-1} B}|b(x)|^{q} d x\right)^{\frac{1}{q}} \leq\left|2^{k} B\right|^{\frac{1}{q}-1} 2^{-\varepsilon k}
$$

and

$$
\int b(x) w(x) d x=0 .
$$

Obviously every $(1, q, w)$-atom is a $(1, q, \varepsilon, w)$-molecule. It is also not hard to see that for fixed $q>1$ and $\varepsilon>0$ there is a constant $C>0$ such that every $(1, q, \varepsilon, w)$ molecule $b$ can be decomposed into a sum

$$
b(x)=\sum_{n=1}^{\infty} \lambda_{n} a_{n}, \quad \sum_{n=1}^{\infty}\left|\lambda_{n}\right| \leq C,
$$

where $\lambda_{n} \in \mathbb{C}, a_{n}$ are $(1, q, w)$-atoms.

The following lemma is easy to prove.

Lemma 3.5 Let $1<q<\infty, \delta, \varepsilon>0$ be such that $\delta>d\left(1-\frac{1}{q}\right)+\varepsilon$. Then there is a constant $C>0$ such that if $b(x)$ satisfies Eq. 3.4 and

$$
\left(\int\left|b(x)\left(1+\frac{\left|x-y_{0}\right|}{r}\right)^{\delta}\right|^{q} d x\right)^{1 / q} \leq \frac{r^{-d+d / q}}{C},
$$

then $b$ is a $(1, q, \varepsilon, w)$-molecule associated with $B\left(y_{0}, r\right)$.

In order to prove Theorem 1.10 we shall use general results about Hardy spaces associated with Schrödinger operators $-\mathcal{L}=\Delta-\mathcal{V}(x)$ with non-negative locally integrable potentials $\mathcal{V}(x)$ which were proved in [11]. We say that a function $\mathbf{a}$ is a generalized $(1, \infty, \mathcal{L})$-atom for the Hardy space $H_{\mathcal{L}}^{1}$ if there is a ball $B=B\left(y_{0}, r\right)$ and a function $\mathbf{b}$ such that

$$
\operatorname{supp} \mathbf{b} \subset B, \quad\|\mathbf{b}\|_{L^{\infty}} \leq|B|^{-1}, \quad \mathbf{a}=\left(I-T_{r^{2}}\right) \mathbf{b} .
$$

Then we say that $\mathbf{a}$ is associated with the ball $B\left(y_{0}, r\right)$. It was proved in Section 6 of [11] that the space $H_{\mathcal{L}}^{1}$ admits atomic decomposition with the generalized $(1, \infty, \mathcal{L})$-atoms, that is, $\|f\|_{H_{\mathcal{L}}^{1}} \sim\|f\|_{H_{\mathbf{a t}, \infty, \mathcal{L}}^{1}}$, where the norm $\|f\|_{H_{\mathbf{a t}, \infty, \mathcal{L}}^{1}}$ is defined as in Eq. 3.1 with $a_{j}(x)$ replaced by the general $(1, \infty, \mathcal{L})$-atoms $\mathbf{a}_{j}(x)$.

Lemma 3.7 There is a constant $C>0$ such that for every a being a generalized $(1, \infty, \mathcal{L})$ atom associated with $B\left(y_{0}, r\right)$ one has

$$
\left|\mathcal{L}^{-1 / 2} \mathbf{a}(y)\right| \leq C r^{1-d}\left(1+\frac{\left|y-y_{0}\right|}{r}\right)^{-d} .
$$


Proof The proof follows from functional calculi (see, e.g., [13]). Note that $\mathcal{L}^{-1 / 2} \mathbf{a}=$ $m_{(r)}(\mathcal{L}) \mathbf{b}$ with $m_{(r)}(\lambda)=r\left(r^{2} \lambda\right)^{-1 / 2}\left(e^{-r^{2} \lambda}-1\right)$ and $\mathbf{b}$ such that supp $\mathbf{b} \subset B\left(y_{0}, r\right)$, $\|\mathbf{b}\|_{L^{\infty}} \leq\left|B\left(y_{0}, r\right)\right|^{-1}$. From [13] we conclude that there is a constant $C>0$ such that for every $r>0$ one has

$$
m_{(r)}(\mathcal{L}) f(x)=\int_{\mathbb{R}^{d}} m_{(r)}(x, y) f(y) d y,
$$

with $m_{(r)}(x, y)$ satisfying

$$
\left|m_{(r)}(x, y)\right| \leq C r^{1-d}\left(1+\frac{|x-y|}{r}\right)^{-d} .
$$

Now the lemma can be easily deduced from Eq. 3.8 and the size and support property of $\mathbf{b}$.

\section{Proof of Theorem 1.10}

For real numbers $n>2, \beta>0$ let

$$
g(x)=(1+|x|)^{-n-\beta}, \quad g_{s}(x)=s^{-n / 2} g\left(\frac{x}{\sqrt{s}}\right) .
$$

One can easily check that

$$
\begin{gathered}
\int_{0}^{t} g_{s}(x) d s \leq C|x|^{2-n}\left(1+\frac{|x|}{\sqrt{t}}\right)^{-2-\beta} ; \\
\int_{r^{2}}^{\infty} g_{s}(x) d s \leq C r^{2-n}\left(1+\frac{|x|}{r}\right)^{-n+2} \text { for } r>0 .
\end{gathered}
$$

Moreover, it is easily to verify that for $1<q<\infty, d\left(1-\frac{1}{q}\right)<\alpha \leq d, \beta>0$ one has

$$
\left\||x|^{\alpha-d}\left(1+\frac{|x|}{\sqrt{t}}\right)^{-d-\beta}\right\|_{L^{q}\left(\mathbb{R}^{d}, d x\right)}=C_{\alpha, \beta} t^{(\alpha-d+d / q) / 2}
$$

and

$$
\int|z-y|^{2-d}\left(1+\frac{|z-y|}{r}\right)^{-\beta}\left(1+\frac{|y|}{r}\right)^{-d+\gamma} d y \leq C r^{2}\left(1+\frac{|z|}{r}\right)^{-d+\gamma+2-\beta}
$$

for $0<\gamma<\beta<2$.

Lemma 4.5 Assume that $V(x)$ satisfies the assumptions of Theorem 1.10. Then for $0<$ $\gamma \leq 2$ and $r>0$ one has

$$
\int_{\mathbb{R}^{d}} V(z)\left(1+\frac{|z-y|}{r}\right)^{-d+\gamma} d z \leq c_{d}^{-1} r^{d-2}\left\|\Delta^{-1} V\right\|_{L^{\infty}}
$$

Proof The left-hand side of Eq. 4.6 is bounded by

$$
\begin{aligned}
\int_{|z-y| \leq r} V(z)\left(\frac{r}{|z-y|}\right)^{d-2} d z & +\int_{|z-y|>r} V(z)\left(\frac{|z-y|}{r}\right)^{-d+2} d z \\
& \leq c_{d}^{-1} r^{d-2}\left\|\Delta^{-1} V\right\|_{L^{\infty}} .
\end{aligned}
$$


Proof of Theorem 1.10 We already have known that the operators $(-\Delta)^{1 / 2} L^{-1 / 2}$ and $L^{1 / 2}(-\Delta)^{-1 / 2}$ are bounded on $L^{1}\left(\mathbb{R}^{d}\right)$. It suffices to prove Eqs. 1.11 and 1.12. Set $\gamma=\frac{1}{10}$ and fix $q>1$ and $\varepsilon>0$ such that $\gamma>d\left(1-\frac{1}{q}\right)+\varepsilon$. Set $w_{0}(x) \equiv 1$. According to the atomic and molecular decompositions (see Section 3) the proof of Eq. 1.11 will be done if we verify that $(-\Delta)^{1 / 2} L^{-1 / 2} \mathbf{a}$ is a multiple of a $\left(1, q, \varepsilon, w_{0}\right)$-molecule for every generalized $(1, \infty, L)$-atom $\mathbf{a}$ with a multiple constant independent of $\mathbf{a}$. Identical arguments can be then applied to show that $L^{1 / 2}(-\Delta)^{-1 / 2} \mathbf{a}$ is a multiple of a $(1, q, \varepsilon, w)$-molecule for a being a generalized atom for the classical Hardy space $H^{1}\left(\mathbb{R}^{d}\right)=H_{-\Delta}^{1}$ with a multiple constant independent of $\mathbf{a}$.

Let $\mathbf{a}=\left(I-K_{r^{2}}\right) \mathbf{b}$ be a generalized $(1, \infty, L)$-atom for $H_{L}^{1}$ associated with $B\left(y_{0}, r\right)$. By Lemma 2.11, since $\int w(x) \mathbf{a}(x) d x=0$, we have

$$
\int(-\Delta)^{1 / 2} L^{-1 / 2} \mathbf{a}(x) d x=0 .
$$

Set

$$
\begin{aligned}
J(x) & =\int_{0}^{\infty} \int_{0}^{t} \iint P_{t-s}(x-z) V(z) K_{s}(z, y)\left(L^{-1 / 2} \mathbf{a}\right)(y) d y d z d s \frac{d t}{t^{3 / 2}} \\
& =\int_{0}^{r^{2}} \int_{0}^{t} \iint \ldots+\int_{r^{2}}^{\infty} \int_{0}^{t / 2} \iint+\int_{r^{2}}^{\infty} \int_{t / 2}^{t} \iint \ldots \\
& =J_{1}(x)+J_{2}(x)+J_{3}(x) .
\end{aligned}
$$

Thanks to Eq. 2.9 and Lemma 3.5 it suffices to show that there is a constant $C_{q}>0$, independent of $\mathbf{a}(x)$ such that

$$
\left\|\left(1+\frac{\left|x-y_{0}\right|}{r}\right)^{\gamma} J(x)\right\|_{L^{q}\left(\mathbb{R}^{d}\right)} \leq C_{q} r^{-d+d / q} .
$$

Applying Lemma 3.7 and Eq. 4.1 with $n=d+1$, we obtain

$$
\begin{aligned}
\left|J_{1}(x)\right| & =\left|\int_{0}^{r^{2}} \int_{0}^{t} \iint P_{t-s}(x-z) V(z) K_{s}(z, y)\left(L^{-1 / 2} \mathbf{a}\right)(y) d y d z d s \frac{d t}{t^{3 / 2}}\right| \\
& \leq C \int_{0}^{r^{2}} \int_{0}^{t} \int P_{t-s}(x-z) V(z) r^{1-d}\left(1+\frac{\left|z-y_{0}\right|}{r}\right)^{-d} d z d s \frac{d t}{t^{3 / 2}} \\
& \leq C \int_{0}^{r^{2}} \int P_{s}(x-z) V(z) r^{1-d}\left(1+\frac{\left|z-y_{0}\right|}{r}\right)^{-d} d z \frac{d s}{\sqrt{s}} \\
& \leq C_{N} \int|x-z|^{1-d}\left(1+\frac{|x-z|}{r}\right)^{-N} V(z) r^{1-d}\left(1+\frac{\left|z-y_{0}\right|}{r}\right)^{-d} d z .
\end{aligned}
$$

Consequently,

$$
\begin{aligned}
& \left|J_{1}(x)\right|\left(1+\frac{\left|x-y_{0}\right|}{r}\right)^{\gamma} \\
& \leq C_{N} r^{1-d} \int|x-z|^{-d+1}\left(1+\frac{|x-z|}{r}\right)^{-N+\gamma} V(z)\left(1+\frac{\left|z-y_{0}\right|}{r}\right)^{-d+\gamma} d z .
\end{aligned}
$$


Therefore, using the Minkowski integral inequality together with Eqs. 4.3 and 4.6, we get

$$
\left\|J_{1}(x)\left(1+\frac{\left|x-y_{0}\right|}{r}\right)^{\gamma}\right\|_{L^{q}(d x)} \leq C r^{-d+d / q} .
$$

In order to estimate $J_{2}(x)$ we use Lemma 3.7 and Eq. 4.1 with $n=d$ to obtain

$$
\begin{array}{r}
\left|J_{2}(x)\right|\left(1+\frac{\left|x-y_{0}\right|}{r}\right)^{\gamma} \\
\leq C \int_{r^{2}}^{\infty}\left(1+\frac{\left|x-y_{0}\right|}{r}\right)^{\gamma} \int_{0}^{t / 2} \iint t^{-d / 2} e^{-c|x-z|^{2} / t} V(z) \\
\times K_{s}(z, y) r^{1-d}\left(1+\frac{\left|y-y_{0}\right|}{r}\right)^{-d} d y d z d s \frac{d t}{t^{3 / 2}} \\
\leq C \int_{r^{2}}^{\infty} \iint t^{(2 \gamma-d-3) / 2} e^{-c|x-z|^{2} / t} V(z) \\
\times|z-y|^{2-d}\left(1+\frac{|z-y|}{\sqrt{t}}\right)^{-N+\gamma} r^{1-d-2 \gamma} \\
\times\left(1+\frac{\left|y-y_{0}\right|}{r}\right)^{-d+\gamma} d y d z d t .
\end{array}
$$

Setting $N=\beta+\gamma$ with $0<\gamma<\beta<2$ and applying the Minkowski integral inequality together with Eqs. 4.4 and 4.6 we conclude that

$$
\begin{aligned}
\| J_{2}(x) & \left(1+\frac{\left|x-y_{0}\right|}{r}\right)^{\gamma} \|_{L^{q}(d x)} \\
\leq & C \int_{r^{2}}^{\infty} \iint t^{-(d+3-2 \gamma-d / q) / 2} V(z) \\
& \times|z-y|^{2-d}\left(1+\frac{|z-y|}{\sqrt{t}}\right)^{-\beta} r^{1-d-2 \gamma}\left(1+\frac{\left|y-y_{0}\right|}{r}\right)^{-d+\gamma} d y d z d t \\
\leq & C \int_{r^{2}}^{\infty} \iint t^{-(d+3-2 \gamma-d / q) / 2} V(z) \\
& \times|z-y|^{2-d}\left(1+\frac{|z-y|}{r}\right)^{-\beta}\left(\frac{\sqrt{t}}{r}\right)^{\beta} r^{1-d-2 \gamma}\left(1+\frac{\left|y-y_{0}\right|}{r}\right)^{-d+\gamma} d y d z d t \\
\leq & C \int r^{-2 d+2+d / q} V(z)\left(1+\frac{\left|z-y_{0}\right|}{r}\right)^{-d+2+\gamma-\beta} d z \\
\leq & C r^{-d+d / q} .
\end{aligned}
$$


By Lemma 3.7 and Eq. 4.1 with $n=d$, we have

$$
\begin{aligned}
\left|J_{3}(x)\right| \leq & C \int_{r^{2}}^{\infty} \int_{\frac{t}{2}}^{t} \iint P_{t-s}(x-z) V(z) t^{-\frac{d}{2}} e^{-\frac{c|z-y|^{2}}{t}} \\
& \times\left(1+\frac{\left|y-y_{0}\right|}{r}\right)^{-d} r^{1-d} d y d z d s \frac{d t}{t^{\frac{3}{2}}} \\
\leq & C_{N} \int_{r^{2}}^{\infty} \iint|x-z|^{2-d}\left(1+\frac{|x-z|}{\sqrt{t}}\right)^{-N} V(z) \\
& \times t^{-\frac{d}{2}} e^{-c|z-y|^{2} / t}\left(1+\frac{\left|y-y_{0}\right|}{r}\right)^{-d} r^{1-d} d y d z \frac{d t}{t^{3 / 2}}
\end{aligned}
$$

Hence,

$$
\begin{aligned}
\left|J_{3}(x)\right|\left(1+\frac{\left|x-y_{0}\right|}{r}\right)^{\gamma} \leq & C \int_{r^{2}}^{\infty} \iint|x-z|^{2-d}\left(1+\frac{|x-z|}{\sqrt{t}}\right)^{-N+\gamma} t^{\gamma} V(z) \\
& \times t^{-\frac{d}{2}} e^{-c^{\prime}|z-y|^{2} / t}\left(1+\frac{\left|y-y_{0}\right|}{r}\right)^{-d+\gamma} r^{1-d-2 \gamma} d y d z \frac{d t}{t^{3 / 2}} .
\end{aligned}
$$

By Minkowski’s integral inequality combined with Eq. 4.3 we arrive to

$$
\begin{aligned}
& \left\|J_{3}(x)\left(1+\frac{\left|x-y_{0}\right|}{r}\right)^{\gamma}\right\|_{L^{q}(d x)} \\
& \leq \int_{r^{2}}^{\infty} \iint t^{(-d+2+d / q) / 2+\gamma-3 / 2} V(z) \\
& \quad \times t^{-d / 2} e^{-c^{\prime}|z-y|^{2} / t}\left(1+\frac{\left|y-y_{0}\right|}{r}\right)^{-d+\gamma} r^{1-d-2 \gamma} d y d z d t .
\end{aligned}
$$

Application of Eq. 4.2 with $n=2 d+1-\frac{d}{q}-2 \gamma$ and then Eq. 4.6 yields

$$
\begin{aligned}
& \left\|J_{3}(x)\left(1+\frac{\left|x-y_{0}\right|}{r}\right)^{\gamma}\right\|_{L^{q}(d x)} \\
& \quad \leq C \iint r^{2-3 d+d / q} V(z)\left(1+\frac{|z-y|}{r}\right)^{-2 d+1+d / q+2 \gamma}\left(1+\frac{\left|y-y_{0}\right|}{r}\right)^{-d+\gamma} d y d z \\
& \quad \leq \int r^{2-2 d+d / q} V(z)\left(1+\frac{\left|z-y_{0}\right|}{r}\right)^{-2 d+1+d / q+3 \gamma} d z \\
& \leq C r^{-d+d / q} .
\end{aligned}
$$

The above inequality together with Eqs. 4.11 and 4.13 gives desired Eq. 4.8 and, consequently, the proof of Eq. 1.11 is complete.

Let us note that in the proof Eq. 1.11 we use only Lemmas 2.11, 3.7, and the upper Gaussian bounds for the kernels. The proof of Eq. 1.12 goes identically to that of Eq. 1.11 by replacing Lemma 2.11 by Lemma 2.13 .

\section{Proof of the Riesz Transform Characterization of $H_{L}^{1}$}

Proof Proof of Corollary 1.13 Assume that $f \in H_{L}^{1}$. Then, thanks to Theorem 1.10, there is $g \in H^{1}\left(\mathbb{R}^{d}\right)$ such that $f=L^{1 / 2}(-\Delta)^{-1 / 2} g$. By the characterization of the classical Hardy 
space $H^{1}\left(\mathbb{R}^{d}\right)$ by the Riesz transforms we have

$$
\frac{\partial}{\partial x_{j}} L^{-1 / 2} f=\frac{\partial}{\partial x_{j}} L^{-1 / 2} L^{1 / 2}(-\Delta)^{-1 / 2} g=\frac{\partial}{\partial x_{j}}(-\Delta)^{-1 / 2} g \in L^{1}\left(\mathbb{R}^{d}\right) .
$$

Conversely, assume that for $f \in L^{1}\left(\mathbb{R}^{d}\right)$ we have $\frac{\partial}{\partial x_{j}} L^{-1 / 2} f \in L^{1}\left(\mathbb{R}^{d}\right)$ for $j=1,2, \ldots, d$. Set $g=(-\Delta)^{1 / 2} L^{-1 / 2} f$. Then by Lemma 2.6, $g \in L^{1}\left(\mathbb{R}^{d}\right)$ and

$$
\frac{\partial}{\partial x_{j}}(-\Delta)^{-1 / 2} g=\frac{\partial}{\partial x_{j}}(-\Delta)^{-1 / 2}(-\Delta)^{1 / 2} L^{-1 / 2} f=\frac{\partial}{\partial x_{j}} L^{-1 / 2} f \in L^{1}\left(\mathbb{R}^{d}\right),
$$

which implies that $g \in H^{1}\left(\mathbb{R}^{d}\right)$. Consequently, by Theorem 1.10, $f \in H_{L}^{1}$. Finally Eq. 1.14 can be deduced from Eqs. 5.1, 5.2, and Theorem 1.10.

Acknowledgments The authors want to thank the referee for her/his comments which improved presentation of the paper.

\section{Appendix}

In the appendix we shall prove that the spaces $H_{\mathcal{L}}^{1}$ and $H_{\mathcal{L}, \max , h}^{1}\left(\mathbb{R}^{d}\right)$ defined in the introduction coincide. The proof goes by standard arguments and we do not use atomic decompositions. Let us note that the proof works in more general settings, e.g. for semigroups satisfying Gaussian bounds on spaces of homogeneous type in the sense of Coifman-Weiss [4].

Since $\left\{T_{t}\right\}_{t>0}$ is a strongly continuous semigroup in e.g. $L^{2}\left(\mathbb{R}^{d}\right)$, one can easily deduce from Eq. 1.2 and the semigroup property that $\left\{T_{t}\right\}_{t>0}$ is a pointwise approximate of the identity, that is,

$$
\lim _{t \rightarrow 0} T_{t} f(x)=f(x) \text { a.e. for } f \in L^{1}\left(\mathbb{R}^{d}\right)+L^{\infty}\left(\mathbb{R}^{d}\right) .
$$

Consequently,

$$
|f(x)| \leq \mathcal{M}_{\mathcal{L}} f(x) \text { a.e. for } f \in L^{1}\left(\mathbb{R}^{d}\right)+L^{\infty}\left(\mathbb{R}^{d}\right) .
$$

Let $f_{n} \in L^{2}\left(\mathbb{R}^{d}\right)$ be a Cauchy sequence in the norm Eq. 1.5 and let $q=$ $\lim _{n \rightarrow \infty}\left\|f_{n}\right\|_{H_{\mathcal{L}}^{1}}$. By virtute of Eq. 6.2 we have $\left\|f_{n}-f_{m}\right\|_{L^{1}\left(\mathbb{R}^{d}\right)} \leq\left\|f_{n}-f_{m}\right\|_{H_{\mathcal{L}}^{1}}$. Hence the sequence $f_{n}$ converges to a unique function $f$ in $L^{1}\left(\mathbb{R}^{d}\right)$. We shall prove that $\mathcal{M}_{\mathcal{L}} f \in L^{1}\left(\mathbb{R}^{d}\right)$ and the convergence of $f_{n}$ to $f$ is also in the $\|\cdot\|_{H_{\mathcal{L}}^{1}}$-norm. To see this take a subsequence $n_{k}$ such that

$$
\left\|f_{n_{k}}-f_{n_{j}}\right\|_{H_{\mathcal{L}}^{1}} \leq 3^{-k} \text { for } j \geq k \geq 1
$$

and write

$$
f=f_{n_{k}}+\sum_{j=k}^{\infty}\left(f_{n_{j+1}}-f_{n_{j}}\right) \text { (convergence in the } L^{1}-\text { norm). }
$$

Obviously, $\left\|\mathcal{M}_{\mathcal{L}} f\right\|_{L^{1}\left(\mathbb{R}^{d}\right)} \leq\left\|f_{n_{k}}\right\|_{H_{\mathcal{L}}^{1}}+\sum_{j=k}^{\infty}\left\|f_{n_{j+1}}-f_{n_{j}}\right\|_{H_{\mathcal{L}}^{1}}$, which gives $\left\|\mathcal{M}_{\mathcal{L}} f\right\|_{L^{1}\left(\mathbb{R}^{d}\right)} \leq q$. Further, by Eqs. 6.4 and 6.3, we have

$$
\left\|\mathcal{M}_{\mathcal{L}}\left(f-f_{n_{k}}\right)\right\|_{L^{1}\left(\mathbb{R}^{d}\right)} \leq \sum_{j=k}^{\infty}\left\|f_{n_{j+1}}-f_{n_{j}}\right\|_{H_{\mathcal{L}}^{1}} \rightarrow 0 \text { as } k \rightarrow \infty .
$$


Thus we have proved that $H_{\mathcal{L}, \max , h}^{1}\left(\mathbb{R}^{d}\right) \subset H_{\mathcal{L}}^{1}$.

Assume now that $f \in H_{\mathcal{L}}^{1}$, that is, $f \in L^{1}\left(\mathbb{R}^{d}\right)$ and $\mathcal{M}_{\mathcal{L}} f \in L^{1}\left(\mathbb{R}^{d}\right)$. By Eq. 1.2 for $t>0$ we have $T_{t} f \in L^{2}\left(\mathbb{R}^{d}\right)$, and so $T_{t} f \in H_{\mathcal{L}, \max , h}^{1}\left(\mathbb{R}^{d}\right)$. We shall prove that

$$
\lim _{t \rightarrow 0^{+}}\left\|\mathcal{M}_{\mathcal{L}}\left(T_{t} f-f\right)\right\|_{L^{1}\left(\mathbb{R}^{d}\right)}=0 .
$$

It is well-known that there are constants $C, c>0$ such that

$$
\left|\partial_{t} T_{t}(x, y)\right| \leq C t^{-1} t^{-d / 2} \exp \left(-c|x-y|^{2} / t\right)
$$

(see e.g., [5], [16, Theorem 6.17], [7] and references therein). We claim that there exists a constant $C_{1}>0$ such that for every $t>0$ and $A>1$ one has

$$
\left\|\sup _{s>A t}\left|T_{t+s} f(x)-T_{s} f(x)\right|\right\|_{L^{1}\left(\mathbb{R}^{d}\right)} \leq C_{1} A^{-1}\|f\|_{L^{1}\left(\mathbb{R}^{d}\right)} .
$$

To prove the claim, we note that for $s>A t$, thanks to Eq. 6.6, we have

$$
\begin{aligned}
\left|T_{t+s}(x, y)-T_{s}(x, y)\right| & =\left|\int_{0}^{t} \partial_{u} T_{s+u}(x, y) d u\right| \\
& \leq C \int_{0}^{t}(t+s)^{-1-d / 2} \exp \left(-c^{\prime}|x-y|^{2} /(t+s)\right) d u \mid \\
& \leq C \int_{0}^{t} s^{-1-d / 2} \exp \left(-c^{\prime}|x-y|^{2} / s\right) d u \mid \\
& \leq C t s^{-1-d / 2} \exp \left(-c^{\prime}|x-y|^{2} / s\right) .
\end{aligned}
$$

Hence,

$$
\sup _{s>A t}\left|T_{t+s}(x, y)-T_{s}(x, y)\right| \leq\left\{\begin{array}{l}
C t(A t)^{-1-d / 2} \text { if }|x-y| \leq \sqrt{A t}, \\
C t|x-y|^{-2-d} \text { if }|x-y|>\sqrt{A t},
\end{array}\right.
$$

and, consequently,

$$
\int_{\mathbb{R}^{n}}\left(\sup _{s>A t}\left|T_{t+s}(x, y)-T_{s}(x, y)\right|\right) d x \leq C_{1} A^{-1},
$$

which implies Eq. 6.7.

We are now in a position to complete the proof of Eq. 6.5. Using Eq. 6.7 we obtain

$$
\begin{aligned}
\left\|T_{t} f-f\right\|_{H_{\mathcal{L}}^{1}} & \leq\left\|\sup _{s>A t}\left|T_{t+s} f-T_{s} f\right|\right\|_{L^{1}\left(\mathbb{R}^{d}\right)}+\left\|\sup _{s \leq A t}\left|T_{t+s} f-T_{s} f\right|\right\|_{L^{1}\left(\mathbb{R}^{d}\right)} \\
& \leq C_{1} A^{-1}\|f\|_{L^{1}}+C\left\|\sup _{s \leq A t}\left|T_{t+s} f-f\right|\right\|\left\|_{L^{1}\left(\mathbb{R}^{d}\right)}+C\right\| \sup _{s \leq A t}\left|T_{s} f-f\right|\|\|_{L^{1}\left(\mathbb{R}^{d}\right)} \\
& \leq C_{1} A^{-1}\|f\|_{L^{1}\left(\mathbb{R}^{d}\right)}+2 C\left\|\sup _{s \leq(A+1) t}\left|T_{s} f-f\right|\right\| \|_{L^{1}\left(\mathbb{R}^{d}\right)} .
\end{aligned}
$$

Fix $\varepsilon>0$ and then take $A=\varepsilon^{-1}$. Clearly,

$$
\sup _{s \leq(A+1) t}\left|T_{s} f(x)-f(x)\right| \leq 2 \mathcal{M}_{\mathcal{L}} f(x) \in L^{1}\left(\mathbb{R}^{d}\right) .
$$

Since $\lim _{t \rightarrow 0} \sup _{s<(A+1) t}\left|T_{s} f(x)-f(x)\right|=0$ a.e., we get Eq. 6.5 from Eqs. 6.8 and 6.9 by applying the Lebesgue dominated convergence theorem. 
Let us finally remark that thanks to the subordination formula and results of [14, Chapter 8] we can adapt the above arguments to prove the equivalence of the definitions of Hardy spaces given by means of the Poisson semigroup $e^{-t \sqrt{\mathcal{L}}}$.

\section{References}

1. Auscher, P., Duong, X.T., McIntosh, A.: Boundedness of Banach space valued singular integral operators and Hardy spaces, Unpublished preprint (2005)

2. Bernicot, F., Zhao, J.: New abstract Hardy spaces. J. Funct. Anal. 255, 1761-1796 (2008)

3. Coifman, R.: A real variable characterization of $H^{p}$. Stud. Math. 51, 269-274 (1974)

4. Coifman, R., Weiss, R.G.: Extensions of Hardy spaces and their use in analysis. Bull. Am. Math. Soc. 83, 569-645 (1977)

5. Coulhon, T., Duong, X.T.: Maximal regularity and kernel bounds: observations on a theorem by Hieber and Prüss. Adv. Differ. Equ. 5, 343-368 (2000)

6. Czaja, W., Zienkiewicz, J.: Atomic characterization of the Hardy space $H_{L}^{1}(\mathbb{R})$ of one-dimensional Schrödinger operators with nonnegative potentials. Proc. Am. Math. Soc. 136(1), 89-94 (2008)

7. Duong, X.T., Yan, L.X.: Duality of Hardy and BMO spaces associated with operators with heat kernel bounds. J. Am. Math. Soc. 18, 943-973 (2005)

8. Dziubański, J., Garrigós, G., Martínez, T., Torrea, J.L., Zienkiewicz, J.: BMO spaces related to Schrödinger operators with potentials satisfying a reverse Hölder inequality. Math. Z. 249, 329-356 (2005)

9. Dziubański, J., Zienkiewicz, J.: Hardy space $H^{1}$ associated to Schrödinger operator satisfying reverse Hölder inequality. Rev. Mat. Iberoam. 15, 279-296 (1999)

10. Dziubański, J., Zienkiewicz, J.: Hardy spaces $H^{1}$ for Schrödinger operators with certain potentials. Stud. Math. 164, 39-53 (2004)

11. Dziubański, J., Zienkiewicz, J.: On Hardy spaces associated with certain Schrödinger operators in dimension 2. Rev. Mat. Iberoam. 28(4), 1035-1060 (2012)

12. Dziubański, J., Zienkiewicz, J.: On isomorphisms of hardy spaces associated with Schrödinger operators. J. Fourier Anal. Appl. 19, 447-456 (2013)

13. Hebisch, W.: A multiplier theorem for Schrödinger operators. Colloq. Math. 60/61, 659-664 (1990)

14. Hofmann, S., Lu, G.Z., Mitrea, D., Mitrea, M., Yan, L.X.: Hardy spaces associated with non-negative self-adjoint operators satisfying Davies-Gafney estimates. Mem. Am. Math. Soc. 214(1007) (2011)

15. Latter, R.H.: A decomposition of $H^{p}\left(\mathbb{R}^{n}\right)$ in terms of atoms. Stud. Math. 62(1), 93-101 (1978)

16. Ouhabaz, E.M.: Analysis of Heat Equations on Domains, London Math. Soc. Mono-graphs, vol. 31, Princeton Univ Press (2005)

17. Semenov, Yu.A.: Stability of $L^{p}$-spectrum of generalized Schrödinger operators and equivalence of Green's functions. Int. Math. Res. Not. 12, 573-593 (1997)

18. Simon, B.: Functional Integration and Quantum Physics, 2nd edn. AMS Chelsea Publishing, Providence (2005)

19. Stein, E.: Harmonic Analysis: Real-Variable Methods, Orthogonality, and Oscillatory Integrals. Princeton University Press, Princeton (1993) 